\title{
Relation of triglyceride stores in skeletal muscle cells to central obesity and insulin sensitivity in European and South Asian men
}

\author{
N.G. Forouhi ${ }^{1}$, G. Jenkinson ${ }^{2}$, E. L. Thomas ${ }^{2}$, S. Mullick ${ }^{1}$, S. Mierisova ${ }^{2}$, U. Bhonsle ${ }^{3}$, P. M. McKeigue ${ }^{1}$, J. D. Bell ${ }^{2}$ \\ ${ }^{1}$ Epidemiology Unit, London School of Hygiene and Tropical Medicine, London, UK \\ ${ }^{2}$ Robert Steiner MRI Unit, MRC Clinical Sciences Centre, Imperial College School of Medicine, Hammersmith Hospital, \\ London, UK \\ ${ }^{3}$ Medical Physics Department, Northwick Park Hospital, Middlesex, UK
}

\section{Abstract}

Aims/hypothesis. To compare the relation between intramyocellular lipid content, central obesity and insulin sensitivity in Europeans and South Asians.

Methods. Cross-sectional study of 40 South Asian and European non-diabetic men matched for age and body mass index. We measured intramyocellular lipid by proton magnetic resonance spectroscopy of soleus muscle, insulin sensitivity by the short insulin tolerance test, per cent body fat by dual-energy x-ray absorptiometry and visceral fat by single-slice computed tomography of the abdomen.

Results. South Asians compared with Europeans had a higher mean per cent body fat $(26.8 \%$ vs $22.5 \%$, $p=0.05$ ) and lower insulin sensitivity (mean \pm SEM $2.4 \pm 0.2$ vs $3.4 \% / \mathrm{min} \pm 0.3, \quad p=0.013)$. Mean ( \pm SEM) intramyocellular lipid content was higher in South Asians than in Europeans $(72.1 \pm 7.5$ vs $53.6 \pm 4.9 \mathrm{mmol} / \mathrm{kg}$ dry weight, $p=0.046)$. In Europeans intramyocellular lipid was correlated with per cent body fat $(r=0.50, p=0.028)$, waist:hip ratio $(r=0.74, p<0.001)$, visceral fat $(r=0.62, p=0.004)$ and insulin sensitivity $(r=-0.53, p=0.016)$. In South Asians intramyocellular lipid was not significantly related to insulin sensitivity or obesity, and the strongest associations of insulin sensitivity were with fasting plasma triglyceride and waist:hip ratio.

Conclusion/interpretation. The association of intramyocellular lipid with insulin sensitivity and obesity in Europeans is consistent with the hypothesis that muscle triglyceride mediates the effect of obesity on insulin sensitivity. The absence of a similar relation of insulin sensitivity to intramyocellular lipid in South Asians suggests that other mechanisms underlie the high insulin resistance observed in this group. [Diabetologia (1999) 42: 932-935]

Keywords Intramyocellular lipid, insulin sensitivity, triglyceride, central obesity, per cent body fat, South Asian, European.
Resistance to insulin-mediated glucose uptake has been implicated in the pathogenesis of Type II (non-insulin-dependent) diabetes mellitus, hypertension and coronary heart disease [1]. Insulin resistance is present in several non-European ethnic groups in

Received: 14 December 1998 and in revised form: 17 March 1999

Corresponding author: N. G. Forouhi MD, Epidemiology Unit, London School of Hygiene and Tropical Medicine, Keppel Street, London, WC1E 7HT, UK

Abbreviations: IMCL, Intramyocellular lipid, ISI insulin sensitivity index, WHR, waist: hip ratio; VFA, visceral abdominal fat area; ${ }^{1} \mathrm{H}-\mathrm{NMR}$, proton nuclear magnetic resonance. which prevalence of' Type II diabetes is higher than in Europeans matched with them for weight, for instance in people of South Asian (Indian, Pakistani, Bangladeshi and Sri Lankan) descent [2]. Insulin resistance is strongly associated with obesity, especially central obesity, but the mechanism of this association is poorly understood. One possibility is that non-esterified fatty acids (NEFA) produced by lipolysis of triglyceride stores in muscle cells block glucose uptake either through substrate competition, as proposed over 30 years ago [3], or through direct inhibition of glucose transport [4].

The relation of insulin sensitivity to intramyocellular lipid (IMCL) has been examined in muscle biopsy 
Table 1. Mean values of clinical and metabolic variables and Pearson's correlation coefficients $(r)$ with intramyocellular lipid in men by ethnic group

\begin{tabular}{|c|c|c|c|c|c|c|c|}
\hline & European $(n=20)$ & & & South Asian $(n=20$ & & & $p^{\mathrm{c}}$ \\
\hline & Mean $^{a}$ & $r$ & $p^{\mathrm{b}}$ & Mean $^{\mathrm{a}}$ & $r$ & $p^{b}$ & \\
\hline Age (years) & $47.8 \pm 1.0$ & 0.45 & 0.048 & $46.3 \pm 1.1$ & 0.01 & NS & NS \\
\hline$\%$ body fat & $22.5 \pm 1.7$ & 0.49 & 0.028 & $26.8 \pm 1.3$ & 0.31 & NS & 0.052 \\
\hline WHR & $0.91 \pm 0.01$ & 0.74 & $<0.001$ & $0.92 \pm 0.01$ & 0.25 & NS & NS \\
\hline Visceral fat area ${ }^{\mathrm{a}}\left(\mathrm{cm}^{2}\right)$ & $113.5(87.1,148.1)$ & 0.62 & 0.004 & $137.2(115.2,163.5)$ & 0.14 & NS & NS \\
\hline Fasting glucose $(\mathrm{mmol} / \mathrm{l})$ & $5.3 \pm 0.1$ & 0.10 & NS & $5.4 \pm 0.1$ & -0.20 & NS & NS \\
\hline 2-h insulin ${ }^{\mathrm{a}}(\mu \mathrm{U} / \mathrm{ml})$ & $10.3(6.9,15.6)$ & 0.15 & NS & $51.4(31.6,83.7)$ & -0.09 & NS & $<0.001$ \\
\hline Fasting triglyceride ${ }^{\mathrm{a}}(\mathrm{mmol} / \mathrm{l})$ & $1.46(1.2-1.8)$ & 0.13 & NS & $1.60(1.3,2.0)$ & -0.08 & NS & NS \\
\hline Fasting NEFA ${ }^{\mathrm{a}}(\mathrm{mmol} / \mathrm{l})$ & $0.29(0.24,0.37)$ & 0.04 & NS & $0.34(0.28,0.41)$ & -0.19 & NS & NS \\
\hline 2-h NEFA ${ }^{\mathrm{a}}(\mathrm{mmol} / \mathrm{l})$ & $0.06(0.04,0.10)$ & 0.01 & NS & $0.05(0.04,0.08)$ & 0.03 & NS & NS \\
\hline Physical activity (1-5 rating) & $2.2 \pm 0.2$ & -0.33 & NS & $2.0 \pm 0.2$ & -0.15 & NS & NS \\
\hline Walking score (1-4) & $3.0 \pm 0.1$ & -0.08 & NS & $2.2 \pm 0.2$ & 0.04 & NS & 0.009 \\
\hline
\end{tabular}

Arithmetic means and SEM are given except where the variable has been log transformed $\left({ }^{a}\right)$, where geometric means and $95 \%$ CIs are shown. ${ }^{\text {b. }}$ : significance level associated with $r$; $: p$ value for ethnic difference in mean values; Exact $p$ values are quoted for $p<0.10$ specimens [5]. Proton nuclear magnetic resonance spectroscopy ( $\left.{ }^{1} \mathrm{H}-\mathrm{NMR}\right)$ now enables non-invasive measurement of IMCL content $[6,7]$. We have used this novel technique to test whether IMCL can account for the relations of insulin sensitivity to obesity and body fat pattern and for the lower insulin sensitivity in South Asians compared with Europeans matched for BMI.

\section{Methods}

Subjects. We selected 40 men (20 South Asian and 20 European) at random from 140 healthy men and women aged 40-55 years who had participated in a larger study on the relation between insulin resistance and body fat distribution (to be published elsewhere). These volunteers were recruited at random from eligible people registered with general practitioners. Exclusion criteria were a history of heart disease, diabetes, drugs affecting insulin resistance or lipids and presence of metal implants. The selection of participants was weighted to achieve a wide range of BMI (18 to $\left.34 \mathrm{~kg} \mathrm{~m}^{-2}\right)$. All participants gave written informed consent and ethical approval for the study was given by Ealing Hospital ethics committee.

Measurements. Height, weight and waist and hip circumference were measured to calculate BMI and waist:hip ratio (WHR). Per cent body fat was measured by dual energy x-ray absorptiometry (DEXA) scan (Hologic 4500). Visceral abdominal fat area (VFA) was measured in the attenuation range -190 to $-30 \mathrm{HU}$ by a single slice (10 $\mathrm{mm}$ thickness) tomogram at L4-L5 (exposure time $2.7 \mathrm{~s}$; exposure factors $160 \mathrm{~mA}$ and $120 \mathrm{kV}$ ). A standard 75-g oral glucose tolerance test was done to measure fasting and 2-h glucose, insulin and NEFA. Insulin sensitivity was measured by the short insulin tolerance test [8], during which the insulin sensitivity index (ISI, \%/min) is derived from linear regression of the rate of fall of log glucose from 3 to $15 \mathrm{~min}$ after a bolus of intravenous insulin $(0.05$ units/kg). Physical activity was assessed by three methods: (1) subjects rated themselves as much less active (i), somewhat less active (ii), about as active (iii), somewhat more active (iv), or much more active (v) compared with others their age; (ii) subjects stated if they participated in any regular sport $(\%$ active), and (iii) how far they walked daily $[<1 / 4$ mile (1), 1/4 to 1 mile (2), 1-3 miles (3), or $>3$ miles (4)].

${ }^{1} H$-NMR spectroscopy. The ${ }^{1} \mathrm{H}-\mathrm{NMR}$ spectra of the left leg soleus muscle were acquired on a $1.5 \mathrm{~T}$ Picker prototype system with participants supine with the left leg immobilised in a 30$\mathrm{cm}$ diameter quadrature bird cage coil. Transverse T1-weighted NMR images (TR 300, TE $30 \mathrm{~ms}$ ) were acquired to determine the placement of the ${ }^{1} \mathrm{H}-\mathrm{NMR}$ voxels, with a slice thickness of $10 \mathrm{~mm}$, a $14 \mathrm{~cm}$ field of view and $128 \times 256$ data matrix. Spectra were obtained using a PRESS (point resolved spectroscopy) sequence with $\mathrm{TE} / \mathrm{TR}=135 / 1500 \mathrm{~ms}$ and an $8 \mathrm{~cm}^{3}$ voxel, using the creatine signal as an internal standard. The muscle spectra were analysed by VARPRO [9]. Peak areas for each signal were obtained and lipid resonances were quantified with reference to total creatine after correcting for $\mathrm{T}_{1}$ and $\mathrm{T}_{2}$. The inter-examination coefficient of variation for IMCL with ${ }^{1} \mathrm{H}-\mathrm{NMR}$ was $13 \%$, and the intra-examination reproducibility was $4 \%$ [9].

Laboratory analyses. Plasma glucose was measured by the oxidase method. Plasma insulin concentration was determined by a microplate-based chemiluminometric assay (Molecular Light Technology Research, Cardiff, UK). Plasma triglyceride and NEFA were measured by commercially available assays (Triglycerides N and NEFA-C, 994-75409, WAKO, Alpha Laboratories, Eastleigh, UK).

Statistical analyses. Skewed variables were log (natural) transformed. Relations between variables were examined by simple (Pearson's) correlation and by multiple linear regression analyses. To facilitate comparison of the strength of association between explanatory variables contributing to ISI, continuous variables were divided by their SD. For these variables the standardised regression coefficient is the coefficient associated 

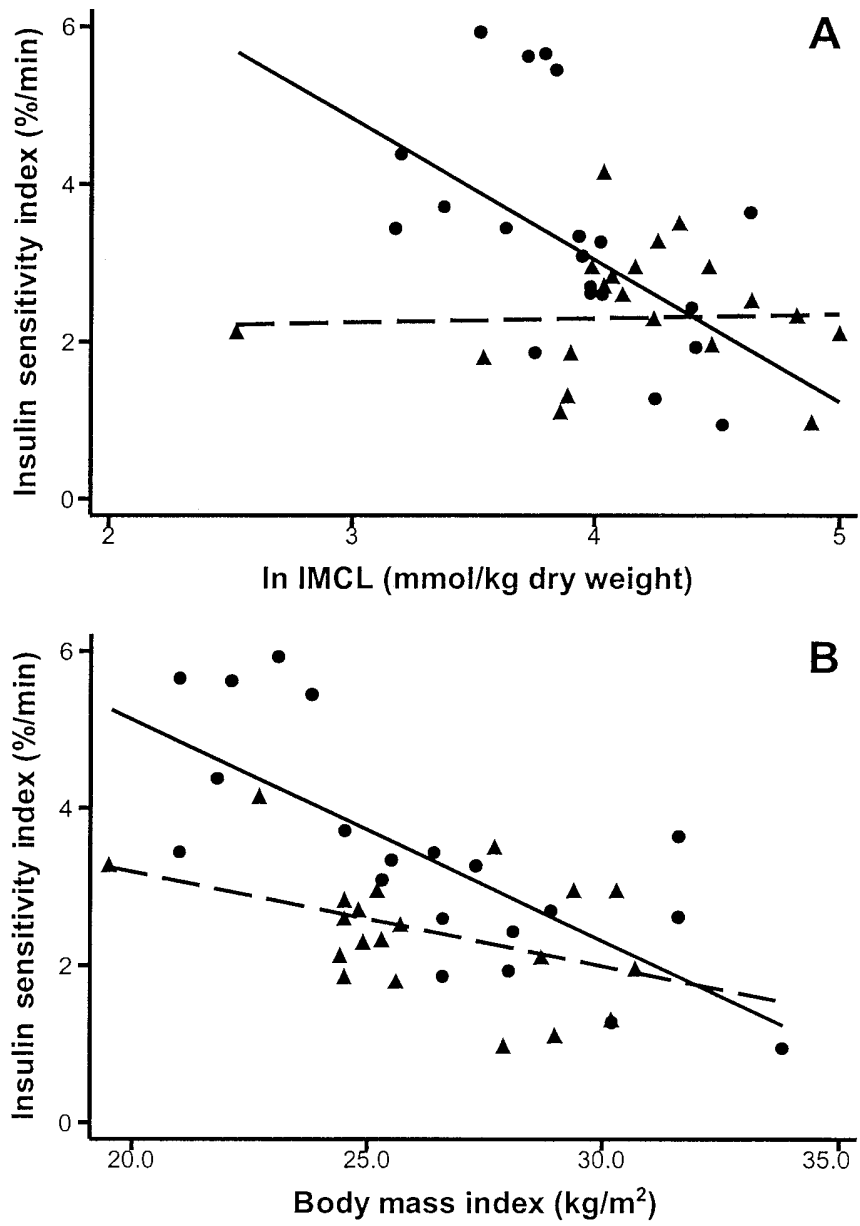

Fig. 1. A. Relation between insulin sensitivity index (ISI) and IMCL content in men of European $(\bullet-)$ and South Asian $(\boldsymbol{\Delta}---)$ descent. In Europeans $(n=20) r=-0.53, p=0.016$; in South Asians $(n=20) r=0.03, p=$ NS. B. Relation between ISI and BMI in men of European ( - ) and South Asian $(\boldsymbol{\Delta}---)$ descent. In Europeans $(n=20) r=-0.75, p<0.001$; in South Asians $(n=20) r=-0.45, p=0.049$

with increase of the variable by one SD. All analyses were done by using STATA (version 5, STATACORP, College station, Texas, USA).

\section{Results}

Mean ( \pm SEM) IMCL was higher in South Asians than in Europeans $(72.1 \pm 7.5 \mathrm{vs} 53.6 \pm 4.9 \mathrm{mmol} / \mathrm{kg}$ dry weight, respectively; $p=0.046)$. The clinical and metabolic measurements of the participants, and their correlation with IMCL are summarised in Table 1. In Europeans IMCL was positively correlated with indices of overall obesity and central obesity, and inversely correlated with ISI (Fig. 1A). In South Asians IMCL was not significantly correlated with any measured variable. European men smoked more and had a higher mean walking score than South Asians, though both groups took little exercise. The distribution of ISI by BMI in the two groups is shown in Fig. 1B.

The strongest (age-adjusted) associations of ISI in Europeans were with: overall obesity ( $\%$ bodyfat: $\beta=-1.52, p<0.001$; BMI: $\beta=-1.03, p=0.001)$, central obesity (VFA: $\beta=-0.79, p=0.005$; WHR: $\beta=-1.0, p=0.034)$, and IMCL $(\beta=-0.8, p=0.058)$. There was no significant association of ISI with plasma lipids among Europeans $(\beta=+0.22,-0.21$ and -0.13 for fasting triglyceride, fasting NEFA and $2 \mathrm{~h}$ NEFA, respectively). Among South Asians ISI was associated with plasma fasting triglyceride $(\beta=-0.72, \quad p<0.001)$ and WHR $(\beta=-0.74$, $p=0.025)$ but not with VFA, IMCL, BMI or per cent body fat.

In age-adjusted multiple regression analyses in Europeans the statistically significant association between ISI and VFA, ISI and percent body fat, and ISI and BMI persisted even after adjustment for IMCL, in contrast to the association between ISI and WHR which was made non-significant when adjusted for IMCL $(\beta=-0.72, p=0.256)$. Among South Asian men the association between ISI and WHR remained significant when adjusted for IMCL $(\beta=-0.80, p=0.022)$. Among European men the significant association between IMCL and WHR, and IMCL and VFA persisted even after adjusting for fasting plasma triglyceride.

The significant relation between ISI and IMCL in Europeans was independent of fasting and 2-h glucose, insulin and NEFA and fasting plasma triglyceride. This association was, however, no longer statistically significant when adjustment was made for overall obesity (percent body fat: $\beta=-0.40, p=0.199$; BMI: $\beta=0.03, p=0.941$ ) or for central obesity (VFA: $\beta=-0.29, \quad p=0.493 ; \quad$ WHR: $\beta=-0.37$, $p=0.494)$. Adjusting for smoking and physical activity did not change the results.

\section{Discussion}

Our findings in Europeans are consistent with the hypothesis that IMCL content modulates insulin sensitivity. We found, like others [7], that the relation in Europeans between ISI and IMCL was independent of fasting and 2-h glucose, insulin and NEFA, and fasting plasma triglycerides. In contrast to other studies where insulin sensitivity was correlated with IMCL independently of BMI and other measures of obesity $[5,7]$, we found that the relation between ISI and IMCL was not independent of obesity and was not stronger than the relation between ISI and obesity [10]. This was the case whether we used creatine or water as the internal standard in ${ }^{1} \mathrm{H}-\mathrm{NMR}$. The reason for these discrepancies is not clear, but is unlikely to be related to data acquisition given that others used similar localisation techniques and have report- 
ed similar reproducibility $[7,10]$. More likely it relates to differences in the population sample, and the larger sample size of our study.

Although in South Asian men IMCL content was higher than in Europeans, IMCL was not significantly related to ISI or indices of obesity. These results are not easily reconciled with the hypothesis that raised IMCL content mediates the associations of central obesity and increased triglycerides with insulin resistance in South Asians. Other physiological mechanisms may mediate impairment of insulin sensitivity in South Asians. For instance increased lipolysis of IMCL stores (modulated by hormone-sensitive lipase) or increased supply of triglycerides from plasma could cause insulin resistance. The strong correlation between plasma triglycerides and ISI observed in South Asians in this study is consistent with this.

In summary, we found a negative correlation between IMCL and insulin sensitivity in Europeans but not in South Asians. To establish definitively whether insulin resistance depends upon muscle cell triglyceride stores will require experimental studies of the effects of dietary and pharmacological interventions that alter muscle triglyceride stores on insulin-mediated glucose uptake. This could open up possibilities for developing new measures to prevent and control insulin resistance and Type II diabetes.

Acknowledgements. This study was supported by a Wellcome Training Fellowship in Clinical Epidemiology for N. G. Forouhi. We acknowledge the Medical Research Council and Picker International for financial support. We thank: J. Kooner for facilitating the study at Ealing Hospital, M. Rowley for help with clinical data collection; the general practitioners who helped with recruitment; B. Svensson for the tomography data; S. Sharp for help with the statistical analysis of the insulin sensitivity data.

\section{References}

1. Reaven GM (1988) Role of insulin resistance in human disease. Diabetes 37: 1595-1607

2. McKeigue PM, Shah B, Marmot MG (1991) Relation of central obesity and insulin resistance with high diabetes prevalence and cardiovascular risk in South Asians. Lancet 337: 382-386

3. Randle PJ, Garland PB, Hales CN, Newsholme EA (1963) The glucose-fatty acid cycle. Its role in insulin sensitivity and the metabolic disturbances of diabetes mellitus. Lancet 1: 785-789

4. Roden M, Price TB, Perseghin G et al. (1996) Mechanism of free fatty acid-induced insulin resistance in humans. J Clin Invest 97: 2859-2865

5. Pan DA, Lillioja S, Kriketos AD et al. (1997) Skeletal Muscle Triglyceride levels are inversely related to insulin action. Diabetes 46: 983-988

6. Schick F, Eismann B, Jung WI, Bongers H, Bunse M, Lutz O (1993) Comparison of localized proton NMR signals of skeletal muscle and fat tissue in vivo: two lipid compartments in muscle tissue. Magn Reson Med 29: 158-167

7. Krssak M, Falk Petersen K, Dresner A et al. (1999) Intramyocellular lipid concentrations are correlated with insulin sensitivity in humans: a ${ }^{1} \mathrm{H}-\mathrm{NMR}$ spectroscopy study. Diabetologia 42: 113-116

8. Hirst S, Phillips DIW, Vines SK, Clark PM, Hales CN (1993) Reproducibility of the short insulin tolerance test. Diabet Med 10: 839-842

9. Rico-Sanz J, Hajnal JV, Thomas EL, Mierisova S, Ala-Korpela M, Bell JD (1998) Intracellular and extracellular skeletal muscle triglyceride metabolism during alternating intensity exercise in humans. J Physiol (Lond) 510: 615-622

10. Stein DT, Szczepaniak LS, Dobbins RL, Snell P, McGarry JD (1998) Skeletal Muscle Triglyceride Stores are Increased in Insulin Resistant States. Proc ISMRM (Sydney, Australia) 388 (Abstract) 